\title{
Comparação Entre Média Geométrica e Médias Ponderadas no Cálculo de Notas em Disciplinas Conjugadas de Química
}

\author{
Anselmo E. de Oliveira, Márlon HF.B. Soares, Aparecido R. de Souza
}

\section{Abstract (Comparison between Geometric and Weighted Means on the Theory/Lab Chemistry Course Scores)}

Theory/Lab courses in General and Physical Chem istry attended by freshmen students of Pharmacy and by second-year students of Food Engineering were evaluated using statistical analysis on the resulting scores calculated by geometric and weighted means as a tool for the allowance of both a better teaching practice and a better teaching process. The obtained results indicated that there were not statistical differences in the scored means although geometric mean scores showed a positive bias resulting in scores systematically higher than the ones for the other weighted means. Since in Chemistry a student needs to have solid background both on theory and lab works, and as a result of summative evaluation geometric mean seems to attend this pre-requisite owed to the fact that the students have to equally dedicate to both lab and theory courses.

\section{Introdução}

\section{Avaliação}

O termo avaliação pode assumir diversos significa dos quando se considera o senso comum e os diversos entendimentos existentes no meio escolar. Nessa perspectiva existe uma gama de teorias a respeito do significado conceitual do termo na tentativa de fundamentá-lo. Em 1967, Michael Scriven contribuiu de maneira significativa para os estudos sobre a avaliação ao propor os conceitos de avaliação somativa e avaliação formativa (Souza, 1998). De acordo com Scriven, esses dois conceitos são funções determinantes da avaliação, pois a função formativa da avaliação pode auxiliar o processo de elaboração de currículos e a função somativa pode julgar o valor de um currículo. As idéias de Scriven sobre avalia-

\footnotetext{
* Instituto de Química, Universidade Federal de Goiás, CP131, 74001-970, Goiânia, GO, Brasil

Correo electrónico: elcana@quimica.ufg.br

Recibido: 4 de abril de 2005; aceptado: 22 de marzo de 2006.
}

ção ainda hoje são utilizadas e adaptadas de alguma maneira para a avaliação escolar

Pode-se dizer que a avaliação relaciona-se em algum momento com a noção de medida. Segundo Bonniol e Vial (Bonniol, 2001) o termo "medir" é a palavra que vem naturalmente à nossa mente quando falamos de avaliação, sendo um termo solidamente ancorado nas mentalidades escolares e na ideologia. Avaliar resultados após uma aplicação de estudos é uma maneira de dar valor a uma atividade realizada.Tal juízo de valor pode ser dado através de notas numéricas ( 0 a 10, ou 0 a 100), ou conceitos alfabéticos equivalentes (A, B, C, D ...), ou ainda conceitos de valor (Bom, Muito Bom, Ótimo, Excelente). Essa prática avaliativa que tem como intuito valorizar um produto, ou ainda classificá-lo de acordo com um valor obtido como retorno, pode, portanto, caracterizar o significado de Avaliação Somativa ou Avaliação Certificativa.

Avaliação formativa é a que se dá durante todo o processo educativo, e procura melhorá-lo. Tanto a avaliação somativa quanto a formativa se dão em diferentes momentos do processo educativo. A somativa ocorre ao final do processo, como resultado e a formativa se dá durante o processo, buscandose um acompanhamento para que se possam fornecer subsídios para o melhoramento da prática pedagógica e do processo pedagógico (Hoffman, 2001).

\section{Médias Aritméticas e Médias Geométricas}

Lee e Colaboradores, 2003, compararam as médias de 165 cursos comportamentais e de ciências sociais nos EUA com as médias que foram atribuídas aos professores pelos alunos, e verificou que os cursos onde os alunos obtinham as maiores médias foram os mesmos onde os professores também receberam as maiores médias. Pôde-se supor que essa correlação deveu-se ao fato de que os melhores professores atraem os melhores alunos, ou que com os melhores professores a instrução é mais efetiva. Uma outra explicação dada foi a de que o aumento nas médias dos alunos nas últimas décadas esteja relacionado à maior difusão do uso da avaliação dos professores pelos alunos. Fato esse que pode levar os 
estudantes a avaliarem a qualidade da instrução baseando-se em professores indulgentes.

No entanto, essa avaliação docente feita pelo corpo discente pode ser vista tanto como uma medida válida da qualidade da instrução (por exemplo Cohen, 1981; Feldman, 1997; Howard, Conway, e Mawxell, 1985; Howard e Maxwell, 1980; 1982; Mars, 1980; 1982; 1984; Marsh e Dunkin, 1992; McKeachie, 1979), quanto posições críticas (por exemplo Chacko, 1983; Dowell e Neal, 1982; Holmes, 1972; Powell, 1977; Snyder e Clair, 1976; Vasta e Sarmiento, 1979; Worthington e Wong, 1979) e posições intermediárias (por exemplo Abrami, Dickens, Perry e Leventhal, 1980). Desse debate surge, naturalmente, o questionamento sobre quais devem ser as medidas aplicadas na avaliação somativa.

Algumas ferramentas estatísticas, como a análise de variância (ANOVA), são bastantes utilizadas pelos pesquisadores na área de educação (Elmore e Woehlke, 1998), embora muitos pesquisadores raramente verifiquem a validade das suposições utilizadas (Keselman e Col., 1998), e a comparação de médias de várias amostras a partir de populações com variâncias diferentes tem sido tema de alguns trabalhos na literatura (por exemplo Kim e Cohen, 1998; Scheffé, 1970; Wang, 1971).

Neste trabalho, a atenção é desviada para a avaliação somativa, realizada em disciplinas conjugadas de química, ou seja, aquelas disciplinas em que a parte teórica e a parte prática são freqüentadas simultaneamente, havendo apenas uma nota final ao término do curso. $\mathrm{O}$ principal método de avaliação somativa, nesses casos, é a atribuição de pesos para cada uma das atividades, teóricas ou práticas. Nessa atribuição de pesos a parte teórica é geralmente priorizada em detrimento da parte experimental.

$\mathrm{Na}$ atribuição de pesos, o aluno tende a valorizar a atividade cujo esforço resulte num maior aproveitamento em termos de nota, e essa dedicação tende a ser proporcional ao peso dado a cada uma das atividades. Ou seja, ele se dedica mais às atividades teóricas que às atividades práticas desenvolvidas no laboratório de ensino. A estratégia de avaliação comumente utilizada é uma ponderação aplicada com os pesos 80-20, 70-30, 60-40 e 50-50, para teoria e prática, respectivamente. Por exemplo, para o peso 80-20 tem-se que $80 \%$ da nota da disciplina resulta da avaliação da teoria e $20 \%$ da atividade prática, e a avaliação 50-50 é a média aritmética entre a teoria e a prática. No entanto, disciplinas de química de vem proporcionar que o aluno se dedique igualmen- te às duas atividades, teoria e prática, ao invés de privilegiar uma ou outra. A formação do aluno do curso de Química, e de outro curso cujas disciplinas de Química componham o projeto pedagógico, deve contemplar uma sólida formação teórica, além das habilidades características e requeridas no trabalho/pesquisa em um laboratório de química.

Desse modo, a utilização de uma avaliação que proporcione ao aluno igual empenho, tanto nas aulas teóricas quanto nas aulas práticas, deve ser considerada. E isso é o que ocorre quando, ao invés da média ponderada, é usada a média geométrica, MG. Essa média, a qual consiste na raiz quadrada do produto das notas de teoria, T, e prática, $\mathrm{P}$,

$$
\mathrm{MG}=\sqrt{\mathrm{T} \times \mathrm{P}}
$$

leva o aluno, independentemente do conteúdo, da carga horária didática e das diferentes habilidades envolvidas em cada uma das atividades da discipli na, a um empenho mínimo tanto para as atividades teóricas, quanto as práticas. Como a Química é uma ciência também experimental, a utilização da média geométrica evita que uma das atividades, seja ela teórica ou experimental, seja valorizada em detrimento da outra, o que normalmente ocorre quando ponderações ou médias aritméticas são empregadas.

$\mathrm{O}$ presente trabalho avalia, estatisticamente, as notas obtidas quando médias ponderadas e a média geométrica são empregadas em disciplinas conjugadas de Química, com o fim de propor, dentre essas médias, a que melhor reproduz o resultado do empenho do aluno nas diversas atividades teóricas e práticas.

\section{Método}

Foram utilizadas nessa avaliação duas disciplinas conjugadas teoria e prática, sendo uma de Química Geral, QG, oferecida aos alunos do $1^{0}$ ano do curso de Engenharia de Alimentos, turma única, e a outra de Físico-Química, FQ, oferecida aos alunos do $2^{0}$ ano do curso de Farmácia, turmas A e B. Essas duas disciplinas são disciplinas anuais, e foram ofe recidas no ano de 2002 pelo Instituto de Química da Universidade Federal de Goiás. A média geométrica foi aplicada em todas as turmas, e ao final do ano letivo os dados foram utilizados na comparação com as outras médias.

Os diferentes resultados para cada uma das disciplinas foram analisados com base nas médias obti das por cada aluno e turma nos quatro bimestres 
letivos -QG foi avaliada apenas nos três primeiros bimestres, devido ao fato do professor da teoria ter alterado a avaliação no quarto bimestre. Na turma de QG havia 45 alunos matriculados, e na turma de FQ havia 35 alunos na turma A e 36 alunos na turma B. Desses alunos, 03 três de QG, e 06 de FQ não cursaram as disciplinas até o fim, e não foram consi derados nos cálculos das médias de cada turma.

Nos casos em que uma das notas, teoria ou prática, foi menor que um, essa nota, para a média geométrica, foi considerada como sendo igual a um. Senão, mesmo o aluno tendo um baixo rendimento em uma das notas, a aplicação da média geométrica diminuiria sobremaneira o rendimento final, obtido, praticamente, com a segunda nota.

O empenho dos alunos e seu comportamento frente às médias utilizadas foram avaliados utilizando-se da observação participante e de conversas informais com os alunos (Bogdan e Biklen, 1999), considerando-se que os autores estavam diretamente envolvidos com as disciplinas ministradas.

\section{Resultados e Discussão}

\section{Médias e Desvios}

Foram avaliadas quatro médias: a média geométrica, MG, e as médias ponderadas, MP, compostas por $80 \%$ da nota da teoria e $20 \%$ da nota da prática, MP80-20, além de MP70-30 e MP60-40. Cada média foi obtida como

$$
\text { Média do Bimestre }=\frac{1}{\mathrm{~N}} \sum_{\mathrm{i}}^{\mathrm{N}} \operatorname{notas}(\mathrm{i})
$$

sendo essa média a somatória das notas de todos os alunos em cada bimestre, dividida pelo número $N$ de alunos da disciplinas/turma. Esses dados constam da Tabela 1, e pode-se observar que $a$ ) praticamente não existem grandes diferenças entre as quatro médias, e $b$ ) quanto maior a diferença entre o peso da nota da teoria e da nota da prática, menor é essa média ponderada em relação à média geométrica. Esse último item mostra que quando o professor decide, por exemplo, adotar uma média ponderada como MP80-20, a nota média dos alunos é, em média, 0,26 por bimestre menor que a média geo métrica. Esse resultado é contrário à idéia que o professor tem de não utilizar a média geométrica por achá-la punitiva, decidindo aplicar uma média como MP80-20. De acordo com os dadosda Tabela 1, qualquer uma das médias resulta em valores médios praticamente similares. Sendo que, a rigor, as médias geométricas e MP60-40 resultam em melhores valores numéricos para as mesmas notas nas avaliações de teoria e prática. Cabe lembrar que como o maior peso é, geralmente, aplicado à parte teórica -como foi o caso no presente estudo-, aonde normalmente se evidenciam as menores notas, quando essa proporção, entre os pesos, é diminuída, verifica-se um aumento nos valores das médias. De modo contrário, tem-se uma redução nos valores das médias à medida que diminui o peso aplicado à parte prática.

Juntamente com as médias foram avaliadas as somas dos desvios, SD, de cada nota individual obtida com a média geométrica, em relação à nota individual obtida com a média ponderada, para cada bimestre das disciplinas. Essa soma de desvios é dada por

$$
\mathrm{SD}=\sum_{\mathrm{i}}\left(\mathrm{nMG}_{\mathrm{i}}-\mathrm{nMP}_{\mathrm{i}}\right)
$$

sendo $\mathrm{nMG}_{i}$ a nota obtida com a média geométrica e $n M P_{i}$ com a nota ponderada para o aluno $i$. Esses dados constam na Tabela 2. Nesse caso, podem-se avaliar os saldos positivos, ou negativos, de pontos de cada turma em cada bimestre, quando se utiliza a média geométrica ao invés da média ponderada. Para a turma de QG tem-se um saldo positivo de 25,50 pontos quando se adota a média geométrica em comparação com a média ponderada MP80-20, e de 8,75 quando comparada com a MP70-30. Ou seja, 25,50 e 8,75 pontos a mais seriam distribuídos entre os alunos para cada uma das turmas, caso a média geométrica fosse adotada, ao invés dessas médias ponderadas. A exceção fica por conta da MP60-40 para QG que, nesse caso, resultaria numa perda de 8,00 pontos caso a média geométrica fosse adotada.

Já para a turma de FQ, Tabela 2, apenas a turma A teria um pequeno ganho em termos numéricos com a adoção da MP60-40. Para as demais médias e para a turma $\mathrm{B}$, a utilização da média geométrica resultaria num ganho numérico favorável.

Apesar das pequenas diferenças verificadas entre a média geométrica e a média MP60-40, essa média ponderada praticamente não é considerada pelos professores de disciplinas conjugadas. Ou seja, se o professor não adota a média MP80-20, nem a MP70-30, ele tende a adotar a média aritmética. Desse modo, significa que se o professor de qualquer uma das turmas resolve adotar uma média ponderada isso resultará, como verificado, numa menor nota, em média, para os alunos, quando comparadas à média que seria obtida caso a média geométrica fosse empregada. Ou seja, a média geométrica, além 
Tabela 1. Médias Geométricas e Ponderadas, por Bimestre, para as Disciplinas de Química Geral, QG, oferecida aos alunos do $1^{0}$ ano de Engenharia de Alimentos e Físico-Química, FQ, turmas A e B, oferecida para os alunos do $2^{\circ}$ ano de Farmácia.

\begin{tabular}{l|c|c|c|c|c}
\hline \multirow{2}{*}{ Disciplina } & \multirow{2}{*}{ Bimestre } & \multicolumn{4}{|c}{ Médias $^{a}$} \\
\cline { 3 - 6 } & & MG & MP80-20 & MP70-30 & MP60-40 \\
\hline \hline \multirow{2}{*}{ QG $^{b}$} & $1^{\circ}$ & 5,60 & 5,36 & 5,51 & 5,65 \\
\cline { 2 - 6 } & $2^{\circ}$ & 5,44 & 5,51 & 5,52 & 5,52 \\
\cline { 2 - 6 } & $3^{\circ}$ & 4,81 & 4,38 & 4,63 & 4,88 \\
\hline \hline FQ-A & $1^{\circ}$ & 6,38 & 6,13 & 6,24 & 6,36 \\
\hline & $2^{\circ}$ & 5,55 & 5,06 & 5,28 & 5,51 \\
\hline \hline FQ-B & $3^{\circ}$ & 4,17 & 3,87 & 4,03 & 4,19 \\
\hline & $4^{\circ}$ & 4,22 & 4,15 & 4,26 & 4,38 \\
\hline & $1^{\circ}$ & 6,69 & 6,29 & 6,46 & 6,62 \\
\hline $2^{\circ}$ & $3^{\circ}$ & 5,54 & 6,11 & 6,29 & 6,47 \\
\hline
\end{tabular}

${ }^{a}$ soma das notas de todos os alunos em cada bimestre, dividida pelo número de alunos.

${ }^{b}$ o $4^{0}$ bimestre de QG não foi incluído nos cálculos, conforme justificado no texto.

de possibilitar igual empenho dos alunos nas diferentes habilidades exigidas nos cursos de teoria e prática -o que não ocorre nas médias ponderadas, nem na média aritmética-, ainda resulta num melhor rendimento em termos da nota, em comparação com as médias ponderadas.

Essa avaliação pode ser verificada a cada bimestre quando as médias obtidas por aluno são comparadas para cada uma das médias avaliadas. Os dados da Tabela 2 para o primeiro bimestre da turma A de
Físico-Química, podem ser visualizados em termos das diferenças entre a nota obtida com média geométrica, $\mathrm{nMG}$, e as suas respectivas médias ponde radas, para cada um dos 35 alunos dessa turma, conforme a Figura 1. Os pontos acima do eixo das abscissas indicam que o valor numérico da média geométrica é maior que o valor da média ponderada, e os pontos abaixo indicam, naturalmente, o comportamento contrário. Nessa figura pode-se observar que a falta de simetria em relação ao eixo das abscis-

Tabela 2: Somas dos Desvios, SD, por Bimestre, para as Disciplinas de Química Geral, QG, oferecida aos alunos do $1^{\circ}$ ano de Engenharia de Alimentos e Físico-Química, FQ, turmas A e B, oferecida para os alunos do $2^{\circ}$ ano de Farmácia.

\begin{tabular}{l|c|c|c|c}
\hline \multirow{2}{*}{ Disciplina } & \multirow{2}{*}{ Bimestre } & \multicolumn{3}{c}{ SD } \\
\cline { 3 - 5 } & & nMG-nMP80-20 & nMG-nMP70-30 & nMG-nMP60-40 \\
\hline \hline QG & $1^{\circ}$ & 10,21 & 4,10 & $-2,01$ \\
\hline & $2^{\circ}$ & $-2,83$ & $-3,01$ & $-3,19$ \\
\hline \hline FQ-A & $3^{\circ}$ & 18,12 & 7,66 & $-2,80$ \\
\hline & $1^{\circ}$ & 8,80 & 4,66 & 0,53 \\
\hline & $2^{\circ}$ & 17,32 & 9,31 & 1,30 \\
\hline \hline FQ-B & $3^{\circ}$ & 10,19 & 4,82 & $-0,54$ \\
\hline & $4^{\circ}$ & 2,46 & $-1,34$ & $-5,13$ \\
\hline & $1^{\circ}$ & 13,54 & 7,86 & 2,19 \\
\hline $2^{\circ}$ & 15,17 & 8,73 & 2,28 \\
\hline & $3^{\circ}$ & 4,36 & 2,45 & 0,54 \\
\hline
\end{tabular}


sas é uma característica de tendência positiva significativa, (ver seção Comparações Emparelhadas), para a diferença entre nMG e nMP80-20, e entre nMG e nMP70-30 - os pontos para esses dois conjuntos de dados estão, na maioria, acima do eixo das abscissas. Ou seja, pode-se dizer que há um erro sistemático quando essas médias ponderadas são utilizadas, resultando que a maioria dos alunos, 63\%, obtém médias bimestrais sistematicamente menores em relação à média geométrica, com os valores de 8,80 e 4,66 para as somas dos desvios em relação à nMP8020 e nMP70-30, respectivamente, conforme Tabe la 2. Para MP60-40 cerca de 51\% das notas são menores que a média geométrica (acima do eixo das abscissas) mas, nesse caso, não há diferença signifi cativa.

Para a média aritmética (MP50-50), 31\% das notas são maiores e as outras $69 \%$ são iguais à média geométrica. A questão da média aritmética é colocada quando um curso cuja nota média para a aprovação é de, por exemplo, cinco pontos. Isso faz com que o aluno possa ser aprovado como excelente na teoria (nota 10,0 , por exemplo), ou na prática, e como insuficiente na outra habilidade (nota 0,0 , por exemplo).

Como a população de médias de uma turma/disciplina pode não seguir uma distribuição normal, e uma nota média para uma turma em um

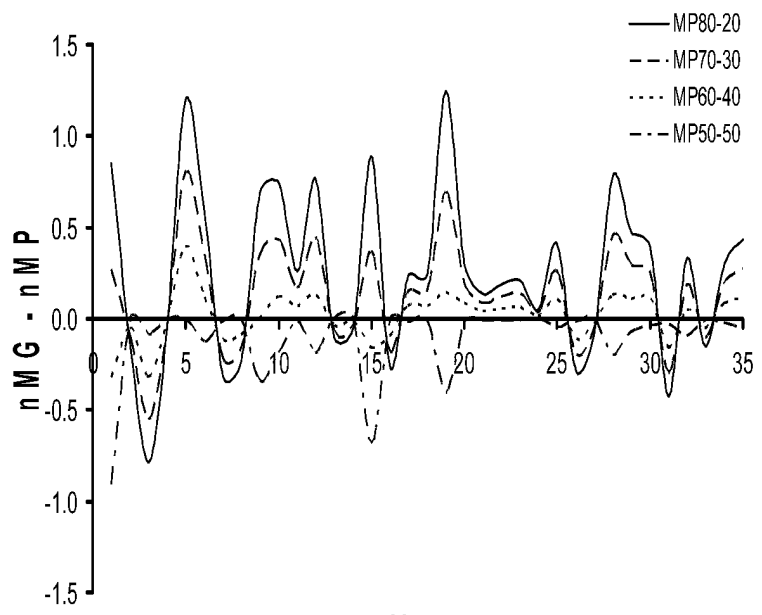

Aluno determinado bimestre pode configurar uma avaliação estatística inadequada, pode-se, então avaliar o histograma das notas médias obtidas para os dados da Figura 1. Este histograma, Figura 2, mostra que a mediana é maior que a média, mas o perfil de distribuição das notas obtidas com as médias geométricas e ponderadas é similar. As medianas valem 6,71, 6,35, 6,48 e 6,60 para MG, MP80-20, MP70-30 e MP60-40, respectivamente. Evidenciando, novamente, que a nota obtida com a média geométrica não é prejudicial ao aluno em termos do valor numérico, e, neste caso, ainda resulta numa maior mediana em relação às notas das demais médias ponderadas.

De acordo com o histograma da Figura 2, as médias apresentam-se bastante correlacionadas umas às outras, conforme pode ser verificado na Figura 3. Desse modo devem apresentar altos valores para os coeficientes de correlação - próximos de 1obtidos por simples ajuste linear. Fazendo-se esse ajuste entre as médias, resultam coeficientes de co rrelação iguais a 0,9884, 0,9946 e 0,9969 para as correl ções entre as notas obtidas com a média geométrica e MP80-20, MP70-30 e MP60-40, respectivamente.

\section{Análise deVariância}

Para fazer comparações entre várias médias, pode-se lançar mão da análise de variância (Box, 1978) para

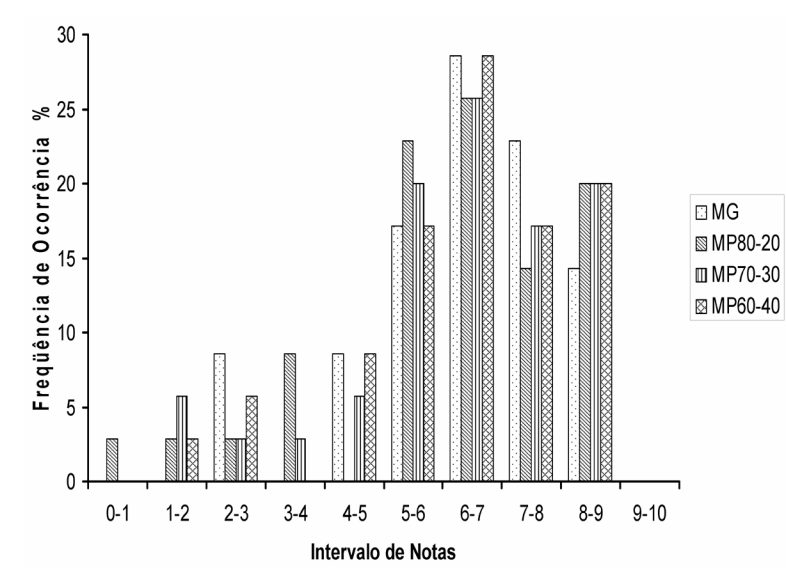

Figura 2. Histograma de notas obtidas com a média geométrica, nMG, e as notas obtidas com as médias ponderadas, nMP, no $1^{\circ}$ bimestre, para os alunos da turma A de Físico-Química.
Figura 1. Gráfico da diferença entre as notas obtidas com a média geométrica, $\mathrm{nMG}$, e as notas obtidas com as médias ponderadas, $\mathrm{nMP}$, no $1^{0}$ bimestre, para os alunos da turma A de Físico-Química.

Octubre de 2006 


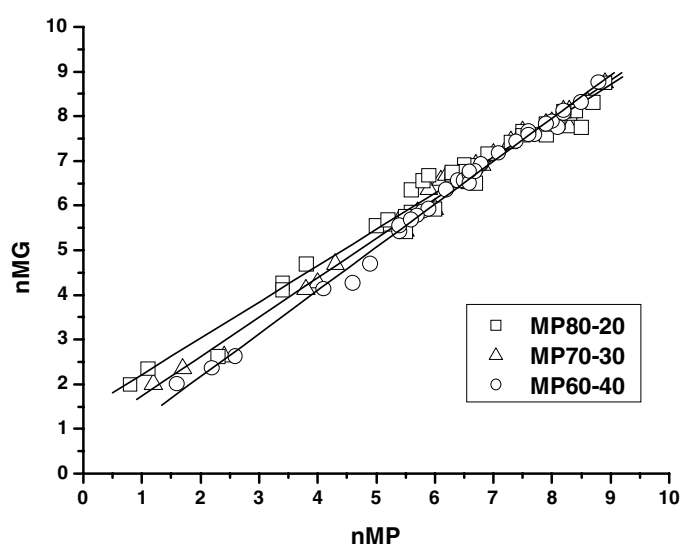

Figura 3. Correlação entre as notas da média geométrica, nMG, e as respectivas notas das médias ponderadas, $\mathrm{nMP}$, no $1^{\circ}$ bimestre, para os alunos da turma A de Físico-Química.

verificar se existem diferenças significativas entre todas as médias. A hipótese nula indica que as médias advêm da mesma população de médias, não havendo diferenças significativas entre todas as médias a serem analisadas.

Avaliando todas as notas dos três primeiros bimestres da disciplina de Química Geral obtém-se um valor de $F=0,16$, bem abaixo do valor tabelado $(F=4,01)$, indicando que hipótese nula é verdadeira, e que não há diferença significativa entre as médias. Para as turmas A e B de Físico-Química, têm-se $F=0,07$ e $F=0,20$, respectivamente, e o valor calculado de $F=3,49$. Nesse caso, a hipótese nula também é verdadeira. Ou seja, não existem diferenças significativas, com $95 \%$ de confiança, entre a média geométrica e as médias ponderadas, demonstrando, estatisticamente, que a média adotada, seja ela geométrica ou ponderada, não resultará em valores médios significativamente diferentes para qualquer uma das três turmas.

Nesse caso, não se pode dizer que a média geométrica é punitiva em relação às demais médias, uma vez que os valores médios obtidos pelas turmas são estatisticamente equivalentes. Cabe afirmar, então, que, quanto aos valores numéricos, qualquer uma das médias pode ser adotada. A diferença fica, desse modo, evidenciada no que se espera em ter mos do aproveitamento e empenho do aluno na sala de aula e no laboratório de ensino, e não na nota em si.

\section{Comparações Emparelhadas}

Para identificar se existem diferenças significativas entre as notas obtidas por duas médias, pode-se calcular o intervalo de confiança da diferença entre essas médias, com a hipótese nula indicando, assim como na seção Análise de Variância, que não há diferença significativa entre essas duas médias. $\mathrm{O}$ intervalo de confiança, D, é dado por (Neto, 2001):

$$
\Delta=\mathrm{d} \mathrm{t}_{\mathrm{v}} \mathrm{S}_{\mathrm{d}} \sqrt{\mathrm{N}}
$$

sendo $\bar{d}$ a média dos desvios entre as notas obtida pelas médias geométrica e ponderada, para cada aluno, em cada turma; $t_{v}$ o valor tabelado de $t \mathrm{com}$ $\checkmark$ graus de liberdade; $s_{d}$ o desvio padrão das médias das variações para cada aluno; e $N$ o número de alunos em cada turma. Se $\Delta$ inclui o zero, significa que a hipótese nula é verdadeira e não há evidência de diferença sistemática entre os resultados das duas médias comparadas.

Comparando a nota obtida com a média geométrica e com as notas das médias MP80-20 e MP70-30, verifica-se que a hipótese nula é falsa, sendo os respectivos intervalos iguais a $[0,08 ; 0,40]$ e $[0,04$; $0,22]$. Nesse caso, há evidência, com $95 \%$ de confiança, que existem diferenças sistemáticas entre as médias. A nota obtida pelo aluno com a média geométrica é sistematicamente maior que as notas obtidas com as médias ponderadas MP80-20 e MP70-30.

Para a comparação entre MG e MP60-40 não há evidência de erro sistemático, sendo as duas médias estatisticamente iguais.

\section{Avaliação do Desempenho}

No início da proposta, encontraram-se resistências iniciais dos alunos, pois os mesmos acreditavam que seriam prejudicados com o modo de calcular a média. A frase a seguir é representativa dessa idéia:

"Essa média (geométrica) é injusta e vai ser difícil passar (na disciplina)"

(Aluno A- Curso FQ)

No decorrer da aplicação, notou-se um aumento da presença dos alunos na sala dos professores, à procura de esclarecer dúvidas, tanto da parte prática quanto da parte teórica. Em anos anteriores, quando da aplicação da média aritmética, a procura era baixa, pois os alunos se saíam bem na parte experimental e praticamente estudavam o mínimo neces sário para serem aprovados na parte teórica. Essa procura aproximou os alunos do professor, fato facilitador do processo ensino-aprendizagem.

Os professores das disciplinas observaram que, 
comparando-se com anos anteriores, os alunos os procuravam mais, tanto nas aulas teóricas quanto nas experimentais, ao invés de tentarem decidir o curso com base apenas nas aulas e estudando pelo livro, sem discutir os temas com os professores.

Em um outro aspecto, após a sugestão dos professores que os alunos fizessem as contas das notas e que as comparassem com as notas obtidas com as médias ponderadas que estavam acostumados, as re clamações com a média geométrica diminuiram porque muitos deles fizeram, de fato, as contas e viram que suas notas eram realmente maiores. Um dos professores, ao comentar as vantagens da média geométrica, em sala de aula, observou um dos alunos balançar a cabeça para o outro colega em sinal de aprovação ao seu resultado obtido com tal média.

Um outro fator importante foi o aumento da presença em sala de aula e em laboratório, principalmente nos final do ano letivo, tradicionalmente esvaziado. O aumento da participação do aluno, teve como consequiência um aumento do interesse pela disciplina, como pode ser constatado na idéia a seguir:

"Eu sei que eu fui bem (na última avaliação) mais ainda assim quero discutir o assunto (tópico da aula prática)"

\section{(Aluno C - Curso FQ)}

Portanto, comparando-se a participação em aula, presença e interesse pela disciplina dos alunos em anos anteriores com o ano de utilização da média geométrica, considerou-se um aumento relevante no empenho. Cabe lembrar que esse trabalho trata de aspectos quantitativos da avaliação, observando-se resultados significantes no empenho. Um estudo qualitativo pode ser realizado, inclusive para detectar como a média geométrica pode auxiliar na melhor forma de se avaliar as disciplinas conjugadas e como esse tipo de média pode melhorar a própria forma de avaliar do professor, o que não é o objetivo deste trabalho.

\section{Conclusões}

Médias geométricas empregadas na avaliação de disciplinas conjugadas em Química resultam em notas estatisticamente equivalentes às obtidas quando médias geométricas são utilizadas e, em alguns casos, os valores numéricos obtidos com a primeira média são sistematicamente maiores que médias ponderadas MP80-20 e MP70-30. Desse modo, a idéia comum entre professores e alunos de que a mé- dia geométrica é prejudicial ao aluno é, na verdade, tão somente uma falsa assertiva. Acrescente-se, ain da, que a média geométrica proporciona ao aluno igual empenho em todas as atividades do curso, sejam essas atividades relacionadas com as atividades teóricas ou práticas. O que torna essa média não discriminatória em relação às diversas avaliações que devem ser feitas nos cursos, atribuindo, a essas avaliações, igual peso/importância, uma vez que todas essas atividades são igualmente importantes na formação do futuro profissional.

Por outro lado, a avaliação somativa, apesar de estar no nível primário de avaliação, ou seja, um estímulo com posterior resposta, pode solidificar bases se funcionar como subsídio do rendimento do aluno em sala de aula, fato mais facilmente observável pelo aluno e pelo professor quando se usa a média geométrica.

\section{Bibliografia}

Abrami, P.C., Dickens, W.J., Perry, R.P., Leventhal, L., Do teacher standards for assigning grades affect students-evaluations of instruction, Journal of Educational Psychology, 72(1), 107-118, 1980.

Bonniol, J.J., Vial, M., Modelos de Avaliação: Textos Fundamentais, Artmed Editora, Porto Alegre, Brasil, 2001.

Bogdan, R.; Biklen, S. Investigação Qualitativa em Educação - Uma Introdução a teoria e aos métodos, Porto Editora, Porto, Portugal, 1997.

Box, G.E.P., Hunter, W.G., Hunter, J.S., Statistics for Experimenters: An Introduction to Design, Data Analysis, and Model Building, Wiley-Interscience, Nova Iorque, EUA, 1978.

Chacko, T.I., Student-ratings of instruction - a function of grading standards, Educational Research Quarterly, 8(2), 19-25, 1983.

Cohen, P.A., Student-ratings of instruction and student-achievement - a meta-analysis of multisection validity studies, Review of Educational Research, 51(3), 281-309, 1981.

Dowell, D.A., Neal, J.A., A selective review of the validity of student-ratings of teaching, Journal of Higher Education, 53(1), 51-62, 1982.

Ellis, L., Burke, D.M., Lomire, P., McCormack, D.R., Student grades and average ratings of instructional quality: the need for adjustment, Journal of Educational Research, 97(1), 35-40, 2003.

Elmore, P.B., Woehlke, P.L., Annual Meeting of the American Educational Research Association, San Diego, EUA, 1998. 
Feldman, K.A., em R.P. Perry, J.C. Smart (editores), Effective teaching in higher education: Research and practice. New York, EUA, 1997.

Hoffman, J.M.L., Avaliar para Promover: As Setas do Caminho, Editora Mediação, Porto Alegre, Brasil, 2001.

Holmes, D.S., Effects of grades and disconfirmed grade expectancies on students evaluations of their instructor, Journal of Educational Psychology, 63(2), 130-133, 1972.

Howard, G.S., Conway, C.G., Maxwell, S.E., Construct-validity of measures of college-teaching effectiveness, Journal of Educational Psychology, 77(2), 187-196, 1985.

Howard, G.S., Maxwell, S.E., Correlation between student satisfaction and grades - a case of mistaken causation, Journal of Educational Psychology, 72(6), 810-820, 1980.

Howard, G.S., Maxwell, S.E., Do grades contaminate student-evaluations of instruction, Research in Higher Education, 16(2), 175-188, 1982.

Keselman, H.J., Huberty, C.J., Lix, L.M., Olejnik, J., Cribbie, R.A., Donahue, B., Kowalchuk, R.K., Lowman, L.L., Petoskey, M.D., Keselman, J.C., Levin, J.R., Statistical practices of educational researchers: an analysis of their ANOVA, MANOVA, and ANCOVA analyses, Review of Educational Research, 68(3), 350-386, 1998.

Kim, S.H., Cohen, A.S., On the Behrens-Fisher problem: a review, Journal of Educational and Behavioral Statistics, 23(4), 356-377, 1998.

Marsh, H.W., The influence of student, course, and instructor characteristics in evaluation of university teaching, American Educational Research Journal, 17(2), 219-237, 1980.

Marsh, H.W., Validity of students evaluations of college-teaching - a multitrait-multimethod analysis, Journal of Educational Psychology, 74(2), 264-279, 1982.
Marsh, H.W., Students evaluations of university teaching - dimensionality, reliability, validity, potential biases, and utility, Journal of Educational Psychology, 76(5), 707-754, 1984.

Marsh, H.W., Dunkin, M.J., em J.C. Smart (editor), Higher education: Handbook of theory and research, Vol. 8, 143-233, Nova Iorque, EUA, 1992.

McKeachie, W.J., Student-ratings of faculty - reprise, Academe-Bulletin of the AAUP, 65(6), 384-397, 1979.

Neto, B. de B., Scarminio, I.S., Bruns, R.E., Como Fazer Experimentos: Pesquisa e Desenvolvimento na Ciência e na Indústria, Editora da Unicamp, Campinas, Brasil, 2001.

Powell, R.W., Grades, learning, and student evaluation of instruction, Research in Higher Education, 7(3), 193-205, 1977.

Scheffé, H., Practical solutions of the Behrens-Fisher Problem, Journal of the American Statistical Association, 65, 1501-1508, 1970.

Snyder, C.R., Clair, M., Effects of expected and obtained grades on teacher evaluation and attribution of performance, Journal of Educational Psychology, 68(1), 75-82, 1976.

Sousa, C.P. Descrição de uma Trajetória na/da Avaliação Educacional, Sistemas de Avaliação Educacional, Série Idéias, n. 30, EPU, São Paulo, Brasil, 199 .

Vasta, R., Sarmiento, R.F., Liberal grading improves evaluations but not performance, Journal of Educational Psychology, 71(2), 207-211, 1979.

Worthington, A.G., Wong, P.T.P., Effects of earned and assigned grades on student-evaluations of an instructor, Journal of Educational Psychology, 71(6), 764-775, 1979.

Wang, Y.Y., Probabilities of type I error of the Welch tests for Behrens-Fisher problem, Journal of the American Statistical Association, 66, 605-608, 1971. 